\title{
Recent advances in activity and travel pattern modelling
}

\author{
Aruna Sivakumar $\cdot$ Abdul Pinjari
}

Published online: 25 April 2012

(C) Springer Science+Business Media, LLC. 2012

These are truly exciting times in the field of travel demand modelling, and it is our pleasure to present this special issue of Transportation at this juncture in the field. On the one hand, activity-based models, that had remained primarily academic until a decade ago, are being embraced worldwide for practical travel forecasting and policy testing. Disaggregate modelling of demand at the level of the individual and the household has become the state of the practice. On the other hand, the complexity of the policy contexts faced by transport planners and decision-makers, and the corresponding challenge to the modelling community, has never been greater. The field is up to the challenge, with developments in multiple directions, on understanding as well as modelling the different nuances of activity and travel behaviour.

- How to enhance our models to incorporate travellers' response to supply capacity constraints?

- What are the different decision rules used by people in scheduling their activities, and how to validate such assumptions in our activity scheduling models?

- Now that we know activity participation drives travel, what are the needs that drive activity participation? And what are the dynamics between the needs that drive activity participation, activity planning, activity generation, and activity scheduling?

- What spatial alternatives do people consider in seeking an activity location, and how to model this choice set of alternatives in a behaviourally realistic manner?

- How best to model traveller choices in the presence of travel time variability (or uncertainty)?

\footnotetext{
A. Sivakumar $(\bowtie)$

Centre for Transport Studies, Imperial College London, South Kensington Campus, London SW7 2AZ, UK

e-mail: a.sivakumar@imperial.ac.uk
}

\author{
A. Pinjari \\ Department of Civil and Environmental Engineering, University of South Florida, 4202 E. Fowler \\ Avenue, ENC 3208, Tampa, FL 33620-5350, USA \\ e-mail: apinjari@usf.edu
}


- How do social networks influence travel behaviour?

- What is the relationship between the different turning points in one's life cycle and long-term mobility decisions?

These are some, by no means exhaustive, lines of recent enquiry in the field. As disparate as these research questions appear, they are all connected by a single, overarching purpose - to enhance our understanding of, and the capability to model, activity-travel behaviour, to produce more realistic and policy sensitive forecasts of travel demand.

This special issue of Transportation brings together a collection of papers addressing some of the above research questions. These papers were presented at the 2012 Annual Meeting of the Transportation Research Board (TRB), in sessions sponsored by the Committee on Travel Behaviour and Values (ADB 10) and the Committee on Travel Demand Forecasting (ADB 40). While this collection may seem very heterogeneous at first glance, a close reading of the papers identifies common underlying motivations viz. the desire to enhance our understanding of travel behaviour, to improve realism in modelling behaviour, and to advance the state of activity-based travel forecasting practice. For instance, the first two papers in this issue, by Zorn et al. and Sammour et al., seek to make improvements to existing activity-based model systems. The former is focused on incorporating the impacts of transit capacity constraints in the San Francisco activity-based travel forecasting model, and the latter on validating the sequence of decision rules used in the ALBATROSS model system from the Netherlands. The third paper, by Nijland et al., is the latest in the series of research papers documenting the development of a new needbased model system in the Netherlands, which seeks to extend the activity-based paradigm to consider the underlying needs. In this paper, the authors stress the importance of modelling the dynamics of activity generation and scheduling with a specific focus on the impact of pre-planned activities. The remaining four papers, while not explicitly linked to a specific activity-based model system, are aimed at improving the behavioural realism of the models embedded in such systems. Yoon et al. seek to improve the specification of choice sets for location choice models, by drawing on Hägerstrand's (1970) concept of the time-space prism. Hu et al. examine different model structures in their ability to capture the behavioural responses to travel time variability, with the objective of improving the modelling of choices in risky situations. The last two papers present empirical results to highlight the importance of issues that are traditionally ignored. Specifically, van de Berg et al. analyze membership and participation in clubs/voluntary associations endogenously with the size and composition of social networks, while Beige and Axhausen examine the relationship between various turning points in life and long-term mobility decisions.

Over the next few paragraphs we present more detailed summaries for each of the seven papers in this special issue.

In the first paper, Zorn, Sall, and Wu address the problem of incorporating the influence of transit capacity constraints and resulting crowding effects on transit ridership assignment. It is not uncommon for transit vehicles in cities like San Francisco to get crowded along high ridership routes. Such capacity constraints can potentially influence traveller behaviour in a variety of ways. For instance, a bus traveller who expects a crowded bus is likely to make one of the following choices: (a) board the same bus at an upstream stop before it gets crowded, (b) wait for the next bus, (c) travel by a different route, (d) switch the mode, time, or destination of travel, or (e) choose not to travel at all. The authors incorporate these effects using a simple yet effective solution. They enable the transit travel times as "variable" and dependent on transit ridership. To do so, they express transit vehicle dwell times (hence traveller waiting times) as a function of boarding, alighting, and 
other crowding metrics using empirical data from automated passenger counters. Now that the transit level of service is dependent on the ridership (and vice versa), they run the transit assignment iteratively to arrive at the equilibrium ridership estimates. Implementing this approach in the San Francisco activity-based model improved the model's ridership predictions, which was otherwise oversubscribing riders onto the most accessible routes beyond their true capacity limitations. Empirical enhancements of this sort represent how our travel forecasting models can be made robust for transit ridership prediction and policy analysis.

The second paper, by Sammour, Bellemans, Vanhoof, Janssens, and Wets, focuses on the validation of rule-based activity-travel forecasting models; specifically the ALBATROSS model system developed in the Netherlands. Rule-based models use a variety of decision rules to mimic individuals' activity scheduling processes, as opposed to modelling the observed decision outcomes directly. However, most validation efforts for these models have focused on comparing predicted and observed outcomes rather than validating the scheduling processes. To address this gap, the authors propose the use of sequence alignment methods (SAM), which are typically used in the field of bioinformatics to assess the degree of similarity between gene sequences. The (dis)similarity is estimated based on the amount of effort required to make two sequences identical. In this paper, the authors propose the use of SAM to validate the work activity scheduling process, which consists of a sequence of decisions including whether to work, the total work duration, number of work episodes, duration of each episode, and start time of each episode. Specifically, they use SAM to compare the sequence of predicted decision outcomes against the sequence of observed outcomes. Application of this method to the work scheduling process in ABLATROSS suggests that such a process-level validation provides additional information to what is known from validating individual decision outcomes. Specifically, the value of using SAM lies in: (a) providing insights on the sequence in which the decisions ought to be modelled, and (b) in identifying the critical decision steps in the work activity scheduling process, which have a higher influence than other steps on the overall prediction performance. Such insights could potentially enhance our understanding of the performance of rule-based activity-travel forecasting models.

The next paper, by Nijland, Arentze and Timmermans, extends a dynamic need-based activity-travel demand model developed by Arentze and Timmermans (2009) by adding the possible effects of planned activities. In particular, the paper addresses the question of how rhythms in need-driving activities are merged with pre-planned activities and events. The proposed model assumes that planned future activities can affect the threshold for conducting an activity, inversely to the number of days between the planned activity and the current day. Data was collected through a web-based survey, both retrospectively and prospectively, regarding activities undertaken and planned. A Bayesian estimation procedure was used to circumvent problems with the atypical and non-smooth likelihood function. The model estimations lead to a wide range of plausible interpretations of the dynamics of activity generation, such as the frequency of undertaking different activity types, preferences for specific days of the week, and the influence of one activity type on another. Planned activities are mostly observed to lower the threshold for conducting activities - this is interpreted to mean either need induction (e.g. a planned social activity might trigger grocery shopping as a preparation for the activity) or time use planning (e.g., if a social activity is planned for the next day, then there is not much time left on that day for maintenance activities such as grocery shopping, so it would be better to execute the latter activities today). While there are some substitution effects (increasing the threshold) noted, most effects of planned activities appear to be complementary (decreasing the 
threshold). The paper also demonstrates how a relatively simple extension of currently used activity diaries can enable the modelling of dynamic activity generation and scheduling processes.

The fourth paper, by Yoon, Deutsch, Chen, and Goulias, addresses a particularly thorny issue related to modelling activity location choices - the specification of choice sets. It is well recognized that misspecification of choice sets can lead to biased parameter estimates and incorrect location choice predictions. The problem is compounded by the large number of location choice alternatives the analyst has to construct the choice set from. This paper provides a behaviourally appealing yet computationally feasible approach to address this issue. The proposed approach starts with identifying discretionary time windows between skeletal activities that are spatially and temporally constrained. For each such time window, Hägerstrand's concepts of time-space prisms are used to identify the potential path areas (PPAs) between the skeletal activities. The PPAs represent the spatial extent that a person can access given the time available, the transport network configuration, and the minimum duration required for pursuing any activity within the time window. Next, the PPAs are overlaid on the spatial organization and the diurnal profile of activity opportunities in the study region to determine the activity opportunities available to the person. These same activity opportunities comprise the feasible choice alternatives for a location choice model. The authors provide compelling empirical illustrations of this approach in the context of the South California region using a variety of commonly used data sources. They analyze how the PPAs (and feasible activity locations) between home and work activity locations are influenced by various factors, such as the amount of time available and flexibility in arrival/departure times etc. The analysis suggests that not only the feasible destination choice sets but also the characteristics of the destination alternatives vary with the above factors. In summary, this paper provides a proof of concept and empirical illustrations of how the concept of time-space prisms can be used to enhance behavioural realism in modelling location choices.

The next paper, by Hu, Sivakumar and Polak, is on the use of non-expected utility theory (non-EUT) approaches to modelling travel choices. Such departures from the more commonly used EUT-based models are gaining increasing interest in the travel modelling community, as a means to capture what is perceived as 'non-rational' behaviour. However, most non-EUT studies, both in transport research and in behavioural economics where the approach originated, are based on stated choice data and laboratory experiments. The authors argue that it is important to analyse these non-EUT models in a revealed preference (RP) context in order to truly understand their value for the travel demand modelling community. Using RP data from a real world experiment on the SR91 corridor in the US, they provide an empirical assessment of non-EUT models for modelling travel behaviour in the presence of travel time variability. Travellers are assumed to choose between a tolled route (a 'certain prospect', in the language of behavioural economics and prospect theory or PT) and an untolled route (an 'uncertain prospect' due to congestion delays). The authors estimate a range of models-including EUT, weighted utility theory, rank dependent utility theory, and PT - and evaluate their performance in terms of goodness of fit, willingness to pay/accept and behavioural implications. The main finding is that there is very little difference in model fit between the EUT and non-EUT models, which questions the value of the non-EUT models of travel time variability in a real-life context. However, the authors acknowledge that this could be a result of poor network performance data. Nevertheless, the take-away idea is that the non-EUT models need to be explored a lot more in a RP context before we can apply them in the real world. 
The authors of the sixth paper, van den Berg, Arentze and Timmermans argue that there has been insufficient research into the different forms of leisure activities, which are inherently very heterogeneous. Specifically, participation in clubs and voluntary associations are often merged with other leisure or social activities despite being more fixed in time, location and company. This paper analyzes membership in clubs/voluntary associations and the participation frequency in club activities. Acknowledging the potential endogeneity between social networks and activity generation, this paper uses a path analysis approach (a special case of structural equation models). The model results are discussed in terms of direct effects, indirect effects, and total (direct + indirect) effects; and four sets of relationships are discussed: (a) relationships between the endogenous variables (social network size, share of relatives in social network, number of clubs/ voluntary associations belonging to, frequency of visits to associations), (b) effects of demographic variables on social network size and composition, (c) effects of demographic variables on the number of clubs or associations, and (d) effects of demographic variables on travel frequency to association activities. One of the findings, among many, is that people get in contact with associations and become members through the interactions within their social networks; which also suggests that there is no substitution effect, but rather complementarity between informal ties and formal memberships.

The last paper in this collection, by Beige and Axhausen, discusses the impacts of key turning points in life, such as personal and familial events (e.g., births, deaths, marriage, separation), on the medium to long-term mobility decisions, such as residential or work location and car ownership. The authors emphasize that such turning points have lasting effects that are important to understand from a policy perspective. Moreover these turning points or 'life course dimensions' are usually not independent from one another. The data used in this paper comes from a retrospective life course survey collected in Switzerland in 2005. The multidimensional life course calendar used to collect this data is a relatively new and under-utilized concept in the transport modelling community. The data is used to estimate a series of binary logit models for the changes in residence, education, employment, car availability and public transport season ticket ownership. The results provide several insights confirming the hypothesis that strong interdependencies exist between the various turning points and long-term mobility decisions during the life course. Changes concerning locations, i.e. the places of residence, education and employment, take place more frequently than changes concerning the ownership of the various mobility tools. At the same time, however, events occur to a great extent simultaneously. As spatial changes take place, the travel behaviour, using mobility tool ownership as a proxy for this behaviour, seems to be reconsidered and altered. The authors conclude on the note that the turning points in life are opportunities to influence behaviour and policies should be designed to utilize these opportunities.

Collectively, the papers in this special issue represent a rapidly growing and widely spawning spectrum of research on understanding and modelling activity-travel behaviour. In addition to their contributions to advancing the state of the practice in activity-based travel forecasting, we would like to highlight a couple of other noteworthy aspects about these papers. The first of these is the issue of endogeneity in travel behaviour, an aspect that has been widely recognized and studied in different contexts. For instance, Zorn et al. address endogeneity between transit ridership (demand) and capacity constraints (supply) via iterative equilibration. Van de Berg et al. handle endogeneity in membership and participation in club activities, and social network size and composition, using path analysis methods. Beige and Axhausen discuss the endogeneity in mobility decisions and life turning points. The modelling of any complex human behaviour is inevitably likely to 
be burdened with the issue of endogeneity, and this is equally true for the new generation of travel demand models based on the activity paradigm. The second point to take away from these papers is that collectively they demonstrate a variety of survey techniques that can be easily exploited to help the translation of this research into practice. For instance, Beige and Axhausen demonstrate the value of the retrospective life course calendar, an idea long known to sociologists, as a means of understanding medium to long-term mobility decisions. Nijland et al. demonstrate the value of a simple extension to the more common activity diary, with the addition of retrospective and prospective questions to capture the dynamics of activity-travel patterns. This is clearly a much less expensive alternative to the panel survey, which has long been considered necessary to model dynamics. While the papers in this special issue are not necessarily the first to propose these survey techniques, the sheer variety of data used in these papers is nevertheless noteworthy.

In closing, we hope you enjoy reading the papers in this special issue as much as we have. Thanks are due to the efforts of Kimberly Fisher, the TRB staff representative to the standing committees responsible for this special issue; Eric Miller, Chair of the TRB Committee on Traveler Behavior and Values (ADB 10); Tom Rossi, Chair of the TRB Committee on Transportation Demand Forecasting (ADB 40); and Martin Richards, the Editor-in-Chief of Transportation, for making this special issue possible. This is the eighth consecutive year of the fruitful partnership between ADB 10, ADB 40, and Transportation in producing a special issue from a select collection of papers presented at the annual TRB meeting. Thanks also to the authors and the reviewers for their contributions to this special issue.

\section{References}

Arentze, T.A., Timmermans, H.J.P.: A need-based model of multi-day, multi-person activity generation. Transp. Res. B 43, 251-265 (2009)

Hägerstrand, T.: What about people in regional science? Pap. Proc. Reg. Sci. Assoc. 24, 7-24 (1970)

\section{Author Biographies}

Aruna Sivakumar is a Lecturer in travel behaviour and demand modelling at the Centre for Transport Studies, Imperial College London. She also leads the transport work streams in multi-disciplinary research projects such as the BP-sponsored Urban Energy Systems Project and the EPSRC sponsored Digital City Exchange Project. Aruna is a Member of the Executive Board of the International Association for Travel Behaviour Research, and is also a member of scientific committees for the TRB and ETC conferences. Aruna holds a PhD and MSc in transportation engineering from the University of Texas at Austin, and her research interests include econometrics, travel behaviour and the role of ICTs, integrated urban system models, and transport policy.

\footnotetext{
Abdul Pinjari is an Assistant Professor in the Transportation Program at the University of South Florida, where he teaches and conducts research in travel demand analysis, activity-based approaches to travel forecasting, freight movement analysis, and choice modeling. He has a $\mathrm{PhD}$ from the University of Texas at Austin, MS from the University of South Florida, and Bachelors of Technology from the Indian Institute of Technology Madras; all degrees in Civil Engineering.
} 